\title{
Habilitación en Conciencia Fonológica para el Trastorno de Aprendizaje de la Lectura: Estudio de un caso
}

\author{
Enabling Phonological Awareness for Reading Learning Disorder: Case Study
}

\section{Jesús Antonio Luna-Padilla ${ }^{\mathrm{a}}$, Ma. de Lourdes Luviano-Vargas ${ }^{\mathrm{b}}$, Ángel Fernando Villalva- Sánchez ${ }^{\mathrm{C}}$}

\begin{abstract}
:
Learning disorder (TA) is conceptualized as learning impairments of academic skills that include: reading, math and writing. Children explicitly trained in phoneme manipulation skills get better results than those who do not. The present study proposes strategies aimed at enabling the phonological path through exercises in phonological awareness using tasks of synthesis, segmentation, addition, omission, substitution of syllables and phonemes, as well as tasks of awareness of rhymes which allow to modify the precision and reading speed and in many other cases reading comprehension. EMB is a girl of 11: .7 years of age, the neuropsychological evaluation showed that EBM meets the diagnostic criteria of AT in its three areas. 120 sessions were held twice a week with a duration of approximately one hour. Synthesis, segmentation, addition, omission and replacement of syllables and phonemes and syllable and phoneme detection tasks in rhymes were designed. EMB showed slight improvements in each of the processes in which it wanted improvement; However, as was observed at the end of the last phase of the treatment, where the grapheme-phoneme correspondence was implemented, EMB managed to have a considerable improvement compared to only the application of phonological tasks.
\end{abstract}

Keywords:

Learning disorder, phonological awareness, reading, writing, intervention.

\section{Resumen:}

El trastorno del aprendizaje (TA) es conceptualizado como afectaciones en el aprendizaje de las habilidades académicas que incluyen: lectura, matemáticas y escritura. Los niños entrenados explícitamente en habilidades de manipulación de fonemas obtienen mejores resultados que aquellos que no. En el presente estudio se proponen estrategias dirigidas a habilitar la ruta fonológica a través de ejercicios en conciencia fonológica utilizando tareas de síntesis, segmentación, adición, omisión, sustitución de sílabas y fonemas, así como tareas de conciencia de rimas las cuales permiten modificar la precisión y velocidad lectora y en muchos otros casos la comprensión lectora. EMB es una niña de 11:.7 años de edad, la evaluación neuropsicológica arrojó que EBM cumple con los criterios diagnósticos de TA en sus tres áreas. Se llevaron a cabo 120 sesiones 2 veces por semana con una duración de una hora aproximadamente. Se diseñaron tareas de síntesis, segmentación, adición, omisión y sustitución de sílabas y fonemas y tareas de detección de sílabas y fonemas en rimas. EMB mostró ligeras mejoras en cada uno de los procesos en los que quería que hubiera mejoría; sin embargo, como se pudo observar al concluir la última fase del tratamiento, donde se implementó la correspondencia grafema-fonema, EMB logró tener una mejoría considerable en comparación que sólo con la aplicación de tareas fonológicas.

Palabras Clave:

Trastorno del aprendizaje, conciencia fonológica, lectura, escritura, intervención.

\section{Introducción}

El trastorno del aprendizaje (TA) es conceptualizado por distintos autores como afectaciones en la adquisición de las habilidades académicas que incluyen: lectura, matemáticas y escritura. La prevalencia del trastorno con sus tres áreas oscila entre $5-15 \%$ en los niños de edad escolar de diferentes lenguas. La

\footnotetext{
a Instituto de Neuropsicología Clínica, https://orcid.org/0000-0003-3378-4991, Email: jesus_lup@ @otmail.com

b Universidad Nacional Autónoma de México, https://orcid.org/0000-0002-4144-9432, Email: 1luviano@unam.mx

c Universidad Nacional Autónoma De México, https://orcid.org/0000-0001-6359-4131, Email: angel.villalva@iztacala.unam.mx
} 
prevalencia en adultos es aproximadamente del 4\% (American Psychiatric Association, 2013). No obstante, estas cifras son obtenidas en censos de Estados Unidos de América, no existen datos certeros en población mexicana, sin embargo, los datos son similares en México, aunque se estima que puede aumentar debido a que hay una gran población sin ser detectada.

Cuando los niños están adquiriendo la lectura generalmente utilizan la ruta indirecta o fonológica (modelo de adquisición de la lectura, Ellis y Young, 1988, para procesar la información y realizar la correspondencia grafema-fonema, por ello es fundamental el uso de habilidades de procesamiento fonológico, que se refieren al uso de la información basada en la estructura del lenguaje oral, para procesar el habla y el lenguaje escrito (Passenger, Stuart \& Terrel, 2003, citados por Herrera et al., 2007)

Por lo tanto, la lectura exige la habilidad de extraer información fonológica, semántica y sintáctica acerca de las palabras (Howell y Manis, 1986 citados por Jiménez y Artiles, 1998) con el objetivo de comprender la información escrita. Debido a esto en la lectura participan diversos procesos como: los perceptivos, que se relacionan con la percepción de las letras y palabras, el proceso fonológico que es la capacidad para manipular e identificar la información fonológica del lenguaje, el proceso léxico, que se relaciona con el reconocimiento de la palabra y su acceso al significado y el proceso sintáctico que permite utilizar un orden para estructurar un conjunto de palabras y por último, el proceso semántico que permite comprender el significado del lenguaje escrito.

Ahora bien, la conciencia fonológica es la habilidad para reflexionar sobre los segmentos fonológicos del lenguaje oral (Tunner, 1989 citado por Jiménez \& Ortiz, 1998). Cuando existe un defecto en la conciencia fonológica se altera el proceso de decodificación del material escrito e impedirá, por tanto, la identificación de las palabras y la comprensión del texto. Se considera que, en las deficiencias en la lectura, las fallas en el procesamiento fonológico son la variable central que explica este tipo de trastornos (Stanovich, 1988; Rueda y Sánchez, 1996; Defior \& Serrano, 2011). Además, desde la perspectiva cognitiva, se menciona que un retraso del proceso fonológico, afecta el desarrollo de las representaciones fonológicas que son las que crean el desarrollo ortográfico. Las consecuencias comunes de este fenómeno son un lento desarrollo de la alfabetización, una deficiente generalización de habilidades de lectura de palabras, de lectura de pseudopalabras y un desarrollo deficiente del deletreo (Snowling \& Griffiths, 2004) aunque no todos los casos se pueden explicar por este factor.

Si bien son destacados los efectos positivos de la intervención, Calero et al. (1999) destacan la ausencia de trabajos en castellano que postulen programas de ejercitación en conciencia fonológica en la educación infantil.

Calero et al. (1999) mencionan que los niños entrenados explícitamente en habilidades de manipulación de los fonemas obtienen mejores resultados que aquellos que no reciben entrenamiento en dichas habilidades (grupo control). A decir de los autores éste programa que ellos presentaron no solo mejoran el desarrollo metafonológico general, sino que lo hace en tareas con mayores exigencias cognitivas y altamente sensibles al aprendizaje lector (Cuadro \& Trías, 2008).

En el caso específico de los trastornos del desarrollo, como el trastorno de aprendizaje, el diagnóstico y tratamiento neuropsicológicos suelen ser muy costosos para la mayoría de la población, por lo que muy pocas familias pueden pagar sus servicios, sobre todo en las regiones rurales. Dada esta situación, la mayoría de los niños con problemas de aprendizaje continúan su educación escolar sin poder tener acceso a este servicio, por lo que se requiere de alternativas que ayuden a salvar estos obstáculos.

En este sentido, en el presente estudio se proponen estrategias dirigidas a habilitar la ruta fonológica a través de ejercicios en conciencia fonológica utilizando tareas de síntesis, segmentación, adición, omisión, sustitución de sílabas y fonemas, así como tareas de conciencia de rimas las cuales permiten modificar la precisión y velocidad lectora y en muchos otros casos la comprensión de lectura.

\section{Descripción del caso}

EMB es una niña de 11 años con 7 meses de edad, la cual cursaba $6^{\circ}$ de primaria al momento de realizar la evaluación inicial. Como antecedentes escolares se refiere que presenta dificultades en la lectura, omite, sustituye y, agrega letras a las palabras, se salta renglones y en ocasiones no comprende lo que lee, en la escritura sustituye palabras y presenta errores ortográficos, en matemáticas acomoda mal las cifras, y tiene dificultades para multiplicar, restar y dividir. También refieren dificultades con la memoria, porque no retiene los conocimientos enseñados en la escuela.

Se realizó una entrevista neuropsicológica infantil. La evaluación neuropsicológica se realizó en FES-Iztacala UNAM, la consta de seis apartados (Datos generales y motivo de consulta, Antecedentes heredofamiliares, Antecedentes personales-familiares no patológicos, Antecedentes Patológicos, Antecedentes gineco-obstetricos y del desarrollo, y Antecedentes escolares) que proporcionan información relevante del desarrollo y aspectos actuales de su desarrollo académico. En los cuales destaca que EMB no presenta datos clínicos para asociar las dificultades a otro aspecto.

Las pruebas que se utilizaron son Escala de Inteligencia de Wechsler para niños IV. (WISC-IV; Wechsler, 2007) y Batería Neuropsicológica para la Evaluación de los Trastornos del Aprendizaje BANETA (Yañez \& Prieto, 2013).

Los resultados de la evaluación neuropsicológica arrojaron que EBM tiene un $\mathrm{Cl}$ de 101 ubicándose en la normalidad y además cumple con los criterios diagnósticos del Trastorno Específico del Aprendizaje Escolar con un desempeño por debajo de lo normal en sus tres áreas lectura, escritura y matemáticas, de acuerdo al Manual Diagnostico y Estadístico para los 
Trastornos Mentales: en su quinta edición (American Psychiatric Association, 2013).

\section{Programa de Intervención en Conciencia Fonológica}

\section{Sesiones}

Se llevaron a cabo 120 sesiones 2 veces por semana, cada sesión con una duración de una hora aproximadamente.

\section{Material}

Se diseñaron tareas de síntesis, segmentación, adición, omisión y sustitución de sílabas y fonemas y tareas de detección de sílabas y fonemas en rimas. Cada tarea constó de 2 actividades y cada actividad contiene 19 ejercicios. El programa se dividió en dos fases, la primera enfocada al Procesamiento Fonológico y la segunda a la Identificación Grafema-fonema.

\section{Fase 1}

Esta primera fase denominada Procesamiento Fonológico, se trabajó en un primer momento con la identificación auditiva de sílabas.

En la primera sección el programa de intervención presenta las siguientes características generales, las cuales se emplean en las sesiones con el fin de mejorar el proceso lector.

- Síntesis de sílabas en palabras

- Segmentación de sílabas en palabras

- Adición de una sílaba en palabras (inicial y final)

- Omisión de una sílaba en palabras (inicial y final)

- Sustitución de una sílaba en palabras (inicial y final).

- Detección de rimas en palabras. (sílaba)

En una segunda sección del programa se trabajó con la identificación auditiva de fonemas.

- Síntesis de fonemas en palabras

- Segmentación de palabra en fonemas

- Adición de un fonema en palabras (inicial y final)

- Omisión de un fonema en palabras (inicial y final)

- Sustitución de un fonema en palabras (inicial y final).

Fase 2

En esta fase se trabajó con la correspondencia grafemas-sílabas, es decir la identificación de las unidades gráficas (letras escritas) y su correspondencia con el sonido (fonemas y sílabas).

En la primera sección se trabajó con la lectura de tarjetas, listas, fichas etc... de sílabas y palabras. Cada actividad constó nuevamente de ejercicios de:

- Síntesis de sílabas en palabras

- Segmentación de sílabas en palabras

- Adición de una sílaba en palabras (inicial y final)
- Omisión de una sílaba en palabras (inicial y final)

- Sustitución de una sílaba en palabras (inicial y final).

- Detección de rimas en palabras. (sílaba)

Finalmente, en la última sección del programa se trabajó con la correspondencia grafema-fonema, es decir la identificación de las unidades grafémicas (letras escritas) y su correspondencia con el sonido (fonemas).

\section{Resultados}

\section{Pretest-Postest}

Con respecto a la puntuación obtenida de EBM Los resultados del coeficiente intelectual se pueden observar en la tabla 1.

Tabla 1.

\begin{tabular}{cccc}
\multicolumn{4}{l}{ Puntajes obtenidos del WISC IV. } \\
\hline Escala & Puntaje LB & $\begin{array}{c}\text { Puntaje } \\
\text { 1a. Fase }\end{array}$ & $\begin{array}{c}\text { Puntaje } \\
\text { 2a. Fase }\end{array}$ \\
\hline ICV & 93 & 93 & 100 \\
IRP & 82 & 82 & 90 \\
IVP & 88 & 83 & 94 \\
IMT & 83 & 88 & 94 \\
CIT & 83 & 83 & 93 \\
\hline
\end{tabular}

Los resultados de la BANETA, fueron favorables tanto en la primera fase, como en la segunda y última fase. Se puede apreciar un incremento en las puntuaciones de ambas fases con respecto a la línea base, los resultados los podemos apreciar en las siguientes gráficas.

\section{Gráfica 1.}

Respuestas de la LB y de la evaluación pretest/postest de Procesamiento Fonológico.

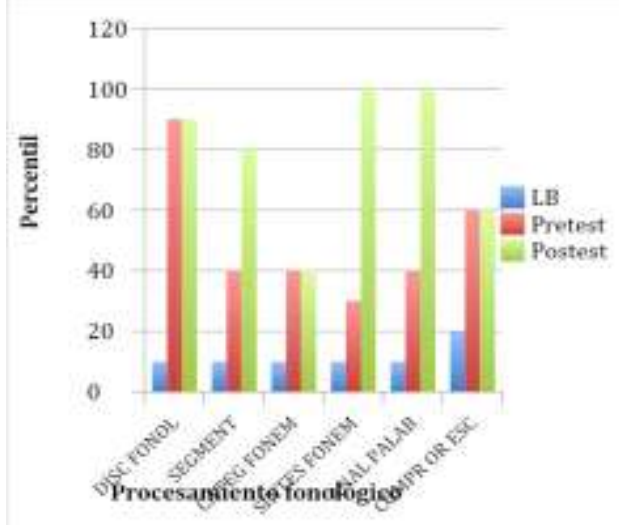

Gráfica 2.

Respuestas de la LB y de la evaluación pretest/postest de Lectura.

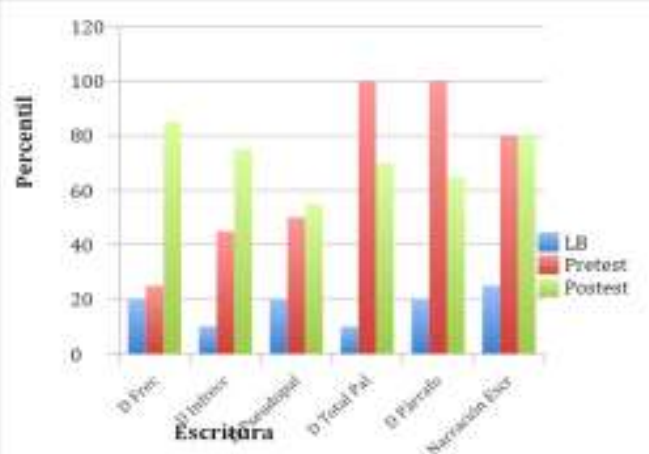


Gráfica 3.

Respuestas de la LB y de la evaluación pretest/postest de Escritura.

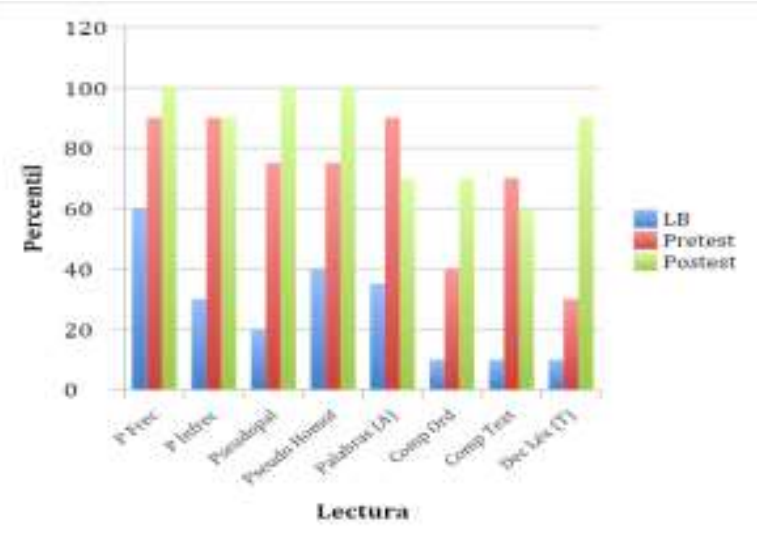

Grafica 4.

Respuestas de la LB y de la evaluación pretest/postest de Matemáticas.

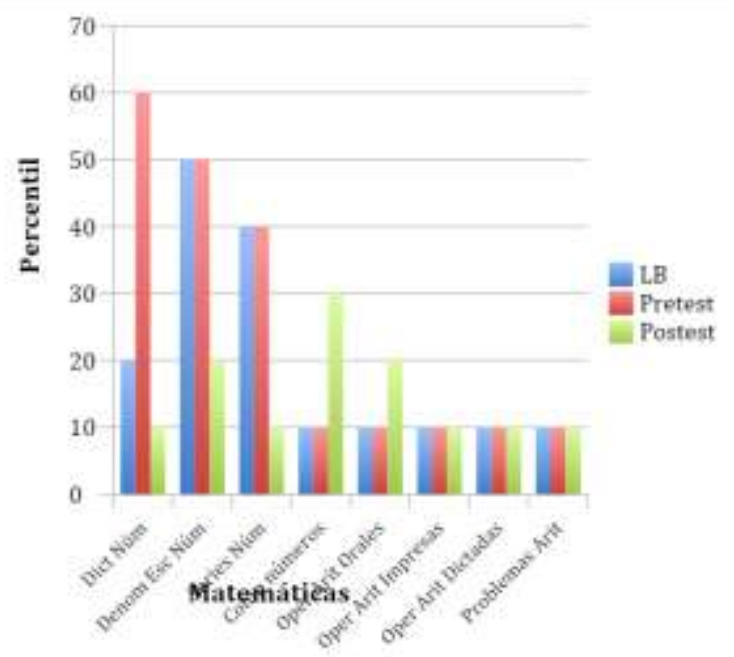

\section{Discusión}

Los datos obtenidos con respecto a la ejecución en las tareas de conciencia fonológica, EMB mostró ligeras mejoras en cada uno de los procesos en los que quería que hubiera mejoría; Sin embargo, como se pudo observar en las gráficas al concluir la última fase del tratamiento, donde se implementó la correspondencia grafema-fonema, EMB logró tener una mejoría considerable en comparación que sólo con la aplicación de tareas fonológicas. Por lo cual, a continuación, se discutirán aspectos de los efectos del tratamiento, los elementos de ayuda para el tratamiento en conciencia fonológica, el uso de estrategias para lograr la comprensión de las tareas y los objetivos alcanzados.

Como en un principio se había señalado, en la lectura participan diversos procesos como el proceso fonológico que es la capacidad para manipular e identificar la información fonológica del lenguaje (Howell y Manis, 1986 citados por Jiménez y Artiles, 1990), las tareas proporcionadas durante la habilitación en el presente estudio estuvieron dirigidas principalmente en este proceso con la intención de mejorar los aspectos lectores, las cuales mostraron la mejoría que la bibliografía ha marcado en las áreas de lectura y escritura principalmente.

Si bien son destacados los efectos positivos de la intervención, en el proceso fonológico, Calero et al., (1999) destacan la ausencia de trabajos en castellano que postulen programas de ejercitación en conciencia fonológica en la educación infantil. En nuestro país se trabaja este trastorno de forma globalizada con otras patologías comórbidas con el trastorno de aprendizaje, sin darle la particularidad que cada caso lo requiere.

\section{Conclusión}

El caso de EBM presentó mejoras después de la implementación de la habilitación en tareas de Conciencia Fonológica, la cual incluye ejercicios de síntesis, segmentación, adición, omisión y sustitución tanto de sílabas como de fonemas, los cuales ayudaron a mejor indiscutiblemente los déficits lectores de EBM presentaba. Cabe señalar que a pesar de que el programa de habilitación no tiene de forma explícita actividades que se enfoquen a la comprensión lectora, se observó en los resultados que EBM mejoró considerablemente, al igual que en la habilidad de escritura. No así en la habilidad de matemáticas, lo cual es un motivo de próximas discusiones.

Es importante comentar que es muy recomendado que el trabajo de ejercitación en los niños con dificultades en el aprendizaje se lleve a cabo en edades y/o grados escolares tempranos, con la finalidad de mejorar no sólo los aspectos académicos, sino también los emocionales y sociales que en muchos casos se ven afectados como parte de las consecuencias secundarias del trastorno.

\section{Referencias}

American Psychiatric Association - APA (2013) Manual Diagnóstico y Estadístico de los Trastornos Mentales - DSM 5. Editorial Médica Panamericana.

Cuadro, A \& Trias, D. (2008). Desarrollo de la conciencia fonémica: Evaluación de un programa de intervención. Revista Argentina de Neuropsicología. 11, 1-8.

Calero, A., Peréz González, R., Maldonado Rico, A., \& Sebastian Gascón, M., E., (1999). Materiales Curriculares para favorecer el acceso a la lectura en Educación Infantil. Madrid: Escuela Española

Ellis, A., \& Young, A. (1988). Human Cognitive Neuropsychology. Londres: LEA.

Herrera, L., Defior, S., \& Lorenzo, O. (2007). Intervención educativa en conciencia fonológica en niños prelectores de lengua materna española y tamazinght. Comparación de dos programas de 
entrenamiento. Infancia y Aprendizaje. 30 (1), 39-54.

Jiménez, J., \& Artiles, C. (1990) Factores predictivos del éxito en el aprendizaje de la lectoescritura. Infancia y Aprendizaje. 49, 21-36.

Jiménez, J., \& Ortiz, M. (1998). Conciencia fonológica y aprendizaje de la lectura: Teoría, evaluación e intervención. España: Editorial Síntesis.

Defior, S., \& Serrano, F. (2011). Procesos Fonológicos Explícitos e Implícitos, Lectura y Dislexia. Revista Neuropsicología, Neuropsiquiatría y Neurociencias. Vol. 11, N 1, 79-94.

Snowling, M. J., \& Griffiths, Y. (2004) Handbook of Children's Literacy, Capítulo 8. USA. Editorial Kluwer Academic Publishers.

Stanovich, K. E. (1988). Explaining the differences between the dyslexic and the garden-variety poor reader: The phonologicalcore variable-difference model. Journal of learning disabilities, 21(10), $590-60$.

Ruerda Sánchez, M., I. \& Sánchez Miguel, E., (1996). Relación entre conocimiento fonológico y dislexia: un estudio instruccional. Cognitiva, 8(2), 215-234.

Yáñez, G., T., \& Prieto, D., M., C., (2013). Batería Neuropsicológica para la Evaluación de los Trastornos del Aprendizaje, BANETA. México: Manual Moderno.

Wechsler, D., (2007). Escala Wechsler de Inteligencia para Niños-IV, Versión estandarizada. Manual Moderno: México. 